Research Article

\title{
Zero Divisor Graph of a Lattice and Its Unique Ideal
}

\author{
Rajendra Deore $\mathbb{D D}^{1}$ and Pramod Tayade $\mathbb{D}^{2}$ \\ ${ }^{1}$ Department of Mathematics, Kavayitri Bahinabai Chaudhary North Maharashtra University, Jalgaon, Jalgaon-425001, India \\ ${ }^{2}$ Department of Mathematics, University of Mumbai, Mumbai-400098, India
}

Correspondence should be addressed to Pramod Tayade; tayade.pramod@yahoo.in

Received 21 July 2019; Accepted 16 September 2019; Published 1 December 2019

Academic Editor: Feng Feng

Copyright (c) 2019 Rajendra Deore and Pramod Tayade. This is an open access article distributed under the Creative Commons Attribution License, which permits unrestricted use, distribution, and reproduction in any medium, provided the original work is properly cited.

Let $L$ be a lattice with the least element 0 . Let $A(L)$ be the finite set of atoms with $|A(L)|>1$ and $\Gamma(L)$ be the zero divisor graph of a lattice $L$. In this paper, we introduce the smallest finite, distributive, and uniquely complemented ideal $B$ of a lattice $L$ having the same number of atoms as that of $L$ and study the properties of $\Gamma(L)$ and $\Gamma(B)$.

\section{Introduction}

Let $L$ be a lattice with the least element 0 . An element $a \in L$ is said to be a zero divisor if there exists a nonzero element $b \in L$ such that $a \wedge b=0$. The set of zero divisors in $L$ is denoted by $Z(L)$. We associate a simple graph $\Gamma(L)$ to $L$ with the vertex set $Z(L)^{*}=Z(L)-\{0\}$; the set of nonzero zero divisors of $L$ and distinct $x, y \in Z(L)^{*}$ are adjacent if and only if $x \wedge y=0$.

In [1], the authors have introduced the notion of coloring in graphs derived from lattices. In [2], the authors associated to any finite lattice $L$ a simple graph $G(L)$ whose vertex set is $Z(L)^{*}$, and two vertices $x$ and $y$ are adjacent if and only if $x \wedge y=0$.

In [3], the authors associated a simple, undirected graph $\Gamma(L)$ with a lattice $L$ with the vertex set $Z(L)^{*}=Z(L)-\{0\}$; the set of nonzero zero divisors of $L$ and distinct $x, y \in Z(L)^{*}$ are adjacent if and only if $x \wedge y=0$. They studied the structure of $\Gamma(L)$ and some basic properties of the zero divisor graph of a lattice. The zero divisor graph of various algebraic structures has been studied by several authors [4-6].

We now give here some preliminaries. Let $(L, \wedge, \vee)$ be a lattice with the least element 0 . An element $a \in L$ is called as an atom if there is no $b \in L$ such that $0<b<a$. The set of all atoms is denoted by $A(L)$. In this paper, we consider lattices with at least two atoms. The lattice $L$ is called atomic if for any $a \in L$, there exists an element $b \in A(L)$ such that $b \leq a$. A nonempty subset $I$ of $L$ is called an ideal if $a, b \in I$ implies that $a \vee b \in I$ and for $l \leq a$ implies $l \in I$. A proper ideal $I$ of $L$ is said to be a prime ideal if $a, b \in L$ and $a \wedge b \in I$ implies $a \in I$ or $b \in I$.

The undefined terms and notations are as in $[2,3]$. Let $G=(V, E)$ be a graph. A graph $G$ is said to be connected if for each pair of distinct vertices $x$ and $y$, there is a finite sequence of distinct vertices $x=v_{1}, v_{2}, v_{3}, \ldots, v_{n}=y$ such that each pair of vertices $\left(v_{k}, v_{k+1}\right) ; 1 \leq k \leq n-1$ is an edge, and such a sequence of vertices is called as a path. The number of edges in a path is the length of a path. For distinct vertices $x$ and $y$ of $G$, let $d(x, y)$ be the length of the shortest path from $x$ to $y \cdot d(x, y)=\infty$ if there is no path from $x$ to $y$ and $d(x, x)=0$. The diameter of a $\operatorname{graph} G$ is $\operatorname{diam}(G)=$ $\sup \{d(x, y):$ where $x$ and $y$ are distinct vertices of $G\}$. The length of a smallest cycle in a graph $G$ is called as girth, and it is denoted by $\operatorname{gr}(G)$ and $\operatorname{gr}(G)=\infty$ if $G$ contains no cycle. The degree of a vertex $v$ in $G$ is the number of edges incident on $v$ which is denoted by $\operatorname{deg}(v)$. The maximum degree vertex of a graph $G, \Delta(G)=\max \{\operatorname{deg}(v): v$ is a vertex of $G\}$. A graph $G$ is said to be bipartite if the vertex set of $G$ is partitioned into two disjoint subsets $P$ and $Q$ such that no edge has both end points in any one of $P$ or $Q$. A complete bipartite graph is a graph whose vertices can be partitioned into two subsets $P$ and $Q$ such that no edge has both endpoints in the same subset, and every possible edge that 
could connect vertices in different subsets is part of the graph. However, in a complete bipartite graph $G$ if $|P|=m$, $|Q|=n$, then it is denoted by $k_{m, n}$. A star graph is a complete bipartite graph $k_{1, n}$. A graph in which each pair of distinct vertices is joined by an edge is a complete graph, and it is denoted by $k_{n}$. A subset $C=\left\{c_{1}, c_{2}, c_{3}, \ldots\right\}$ of $L$ is called a clique in $\Gamma(L)$ if $c_{i}, c_{j}$ are adjacent for all $i, j ; i \neq j$. The clique number $c l \Gamma(L)$ is the cardinality of the maximum possible complete subgraph of $\Gamma(L)$. If the size of a clique is not bounded, then we say $c l \Gamma(L)=\infty$. A (vertex) coloring of $G$ is assigning of colors to the vertices so that adjacent vertices have different colors. The minimum number of colors required to color $G$ is the chromatic number of $G$ and is denoted by $\chi(G)$.

\section{Diameter and Line Graph on Four Vertices}

Lemma 1. Let $L$ be a lattice with the least element 0 and $A(L)$, the set of atoms in $L$. If $|A(L)| \geq 2$, then $A(L) \subseteq Z(L)^{*}$.

Proof. Let $A(L)=\{a, b\}$. Then, $0<a, b$ and $a \wedge b=0$.

Proposition 1. A connected graph on four vertices with vertex set $V(L)=\left\{a_{1}, a_{2}, a_{3}, a_{4}\right\}$ and edges $a_{1}-a_{2}, a_{2}-a_{3}$, $a_{3}-a_{4}$ cannot be realised as $\Gamma(L)$.

Proof. On the contrary, suppose that $L=\left\{0, a_{1}, a_{2}, a_{3}, a_{4}, 1\right\}$ is the required lattice, it must be closed under meet. In particular, $a_{1} \wedge a_{4} \in L$. We have the following cases:

Case 1: $a_{1} \wedge a_{4}=a_{1}$ implies that $a_{1} \wedge a_{3}=\left(a_{1} \wedge a_{4}\right) \wedge$ $a_{3}=0$

Case 2: $a_{1} \wedge a_{4}=a_{2}$ implies that $a_{1} \wedge a_{4}=\left(a_{1} \wedge a_{4}\right) \wedge$ $a_{1}=\left(a_{2}\right) \wedge a_{1}=0$

Case 3: $a_{1} \wedge a_{4}=a_{3}$ implies that $a_{1} \wedge a_{4}=\left(a_{1} \wedge a_{4}\right) \wedge$ $a_{1}=a_{3} \wedge a_{1}=0$

Case 4: $\quad a_{1} \wedge a_{4}=a_{4}$ implies that $a_{2} \wedge a_{4}=a_{2} \wedge$ $\left(a_{1} \wedge a_{4}\right)=0$

In each of the above cases, we get a contradiction. Thus, we have $a_{1} \wedge a_{4}$ is not in $L$. Hence, $L$ is not a lattice.

Proposition 2 ([3], Proposition 1.4). If $L$ is a lattice, then $\operatorname{diam}(\Gamma(L)) \leq 3$.

Theorem 1. Let $L$ be a lattice with the least element 0. If $\Gamma(L)$ contains a cycle, then $\operatorname{gr}(\Gamma(L) \leq 4$.

Proof. Assume that $\Gamma(L)$ contains the smallest cycle $C_{n}$ : $a_{1}-a_{2}-a_{3}-\cdots-a_{n}-a_{1}$ of length $n$. If $n \leq 4$, then we are done. Suppose that $n \geq 5$. Therefore, $a_{1} \wedge a_{4} \neq 0$. We have the following cases for consideration:

Case 1: if $a_{1} \wedge a_{4}=a_{1}$, then $a_{1}-a_{2}-a_{3}-a_{1}$ is a cycle of length three smaller than $n(\geq 5)$. Similarly, $a_{1} \wedge a_{4}=a_{4}$.

Case 2: if $a_{1} \wedge a_{4}=a_{2}$, then $a_{n}-a_{2}-\cdots-a_{n-1}-a_{n}$ is a cycle of length $n-1$, smaller than $n(\geq 5)$.

Case 3: if $a_{1} \wedge a_{4}=a_{3}$, then it is similar to Case 2 .
From Cases 1, 2, and 3, we conclude that $a_{1} \wedge a_{4} \neq a_{1}$, $a_{2}, a_{3}$, and $a_{4}$. However, $a_{2}-a_{1} \wedge a_{4}-a_{3}-a_{2}$ is a cycle of length 3 , smaller than $n(\geq 5)$, a contradiction to our assumption. Hence, $\operatorname{gr}(\Gamma(L)) \leq 4$.

Let $(L, \wedge, \vee)$ be a finite bounded lattice and $a \in L$. Then, we define $[a] \downarrow=\{x \in L: x \leq a\}, \quad[a] \uparrow=\{y \in L: a \leq y\}$ as given in Definition 3.6 of [2]. The base of $k \in L$ is denoted by $B(k)$ and is defined to be the set of all atoms $a$ of $L$ with $a \leq k$ and $B(k)^{c}=A(L) \backslash B(k)$.

Lemma 2 ([2], Lemma 5.7). Let L be a finite bounded lattice and $a, b \in L$ such that $B(a)=B(b)$. Then, $\operatorname{deg}(a)=\operatorname{deg}(b)$.

Following example shows that the converse of the above lemma is not true.

Example 1. For lattice $L=\left\{0, a_{1}, a_{2}, b, 1\right\},\left\{a_{1}\right\}=B\left(a_{1}\right) \neq$ $B\left(a_{2}\right)=\left\{a_{2}\right\}$ and $\operatorname{deg}\left(a_{1}\right)=\operatorname{deg}\left(a_{2}\right)$ in $\left(\Gamma\left(a_{1} \vee a_{2}\right]\right)$.

\section{Unique Ideal of Lattices}

Lemma 3. Let $L$ be a lattice with a nonempty subset $S=$ $\left\{s_{1}, s_{2}, \ldots, s_{n}\right\}$. Then, $I=(S]=\left\{l \in L: l \leq s_{1} \vee s_{2} \vee, \ldots, \vee s_{n}\right\}$ is the smallest ideal of $L$ containing $S$.

An Ideal of a lattice $L$ is a sublattice but not conversely.

Theorem 2. Let $L$ be a nondiamond lattice with the least element 0 and having a finite set of atoms. Then there exists a finite, distributive, and uniquely complemented ideal $B$ of $L$ such that $A(L)=A(B)$.

Proof. Let $A(L)=\left\{a_{1}, a_{2}, a_{3}, \ldots, a_{n}\right\}$ be the set of atoms of $L$. Let $B=(A(L)]=\left[1_{J}\right] \downarrow$, where $1_{J} \neq a_{1} \vee a_{2} \vee \cdots \vee$ $a_{k-1} \vee a_{k+1} \cdots \vee a_{n}, 1_{J}=a_{1} \vee a_{2} \vee \cdots \vee a_{n}$ is the largest element of $B$ which is a locally maximal element of a lattice $L$ and 0 is the least element. By Lemma 3, $B$ is finite and is the smallest ideal of $L$ containing all atoms of $L$.

Corollary 1. Let $L$ be a lattice with the least element 0 and having a finite set of atoms. Then, $B$ is a finite, distributive, and uniquely complemented sublattice of $L$.

Corollary 2. Let $L$ be a lattice with the least element 0 and has a finite set of atoms. Then,

(a) $Z(B)^{*}=B-\left\{0,1_{J}\right\}$

(b) $Z(B) \subseteq Z(L)$

Proof. (a) For $x \in Z(B)^{*}, x=a_{1} \vee a_{2} \vee \ldots \vee a_{k-1} \vee a_{k+1} \ldots \vee$ $a_{n}, x \wedge a_{k}=0$. (b) For $\quad x \in Z(B), x=a_{1} \vee a_{2} \vee \cdots \vee a_{k-1} \vee$ $a_{k+1} \cdots \vee a_{n}, x \wedge a_{k}=0, x \in Z(L)$. If $x=0$, then $x \in Z(L)$.

Corollary 3. Let $L$ be a lattice with least element $0, A(L)=$ $\left\{a_{1}, a_{2}, a_{3}, \ldots, a_{n}\right\}$ be the set of all atoms of $L$, and a unique ideal $B=\left[1_{J}\right] \downarrow$ of L. Let $1_{J}$ and 1 be the largest elements of $B$ and $L$, respectively. Then,

(a) If $1_{J} \leq x<1$, then $x$ has no complement in $L$ 
(b) If $b<1_{J}$, then $b$ has a complement in $B$ but has no complement in $L$

Corollary 4. ([2], Corollary 5.11). Let a be an element of $L$ such that $B(a)=A(L)$ and a be comparable with all elements of $L$. Then, $G(L)=G([a] \downarrow)$.

Theorem 3. Let $L$ be a lattice with the least element 0 and having a finite set of atoms. If $B$ is the unique ideal, then $\Gamma(B)$ is the induced subgraph of $\Gamma(L)$.

Proof. Proof follows by Corollary 2 and Corollary 4 .

Theorem 4. Let $L$ be a lattice with the least element 0 and having a finite set of atoms and $B$ be its unique ideal. Then, $\Gamma(B)$ is the n-partite induced subgraph of $\Gamma(L)$.

Proof. Let $A(L)=\left\{a_{1}, a_{2}, \ldots, a_{n}\right\}, n \geq 2$. Then, $A(B)=\left\{a_{1}\right.$, $\left.a_{2}, \ldots, a_{n}\right\}$ is the set of atoms in a finite sublattice $B$. Therefore, $B-\{0\}=\left(\left[a_{1}\right] \uparrow\right) \cup\left(\left[a_{2}\right] \uparrow\right) \cup \cdots \cup\left(\left[a_{n}\right] \uparrow\right)$. There is no adjacency between any two arbitrary elements in $\left[a_{i}\right] \uparrow$, for all $1 \leq i \leq n$. If $x \in\left(\left[a_{i}\right] \uparrow \cup\left[a_{k}\right] \uparrow\right)$ for some $1 \leq i, k \leq n$, then there is no adjacency between $x$ and both elements $a_{i}$ and $a_{k}$. Thus, there exist disjoint subsets $B_{i}$ of $B-\{0\}, B-$ $\{0\}=B_{1} \cup B_{2} \cdots \cup B_{n}$. This shows that $\Gamma(B)$ is $n$-partite.

Theorem 5. Let $L$ be a finite lattice with $A(L)=\{a, b\}$. $a=$ $x_{0}<x_{1}<x_{2}<x_{3}<\cdots<x_{n}, B=(b \vee a]=B_{0}$ and $B_{i}=\left(b \vee x_{i}\right]$. Then,

(1) $A(B)=A\left(B_{i}\right)$ for all $1 \leq i \leq n$

(2) $B \subset B_{1} \subset B_{2} \subset B_{3} \subset \cdots \subset B_{n}$

(3) $\Gamma(B) \subset \Gamma\left(B_{1}\right) \subset \Gamma\left(B_{2}\right) \subset \Gamma\left(B_{3}\right) \subset \cdots \subset \Gamma\left(B_{n}\right)$

(4) $\Gamma(L)=K_{1, n}$

(5) $\operatorname{gr}(\Gamma(L))=\operatorname{gr}(\Gamma(B))=\infty$

Theorem 6. Let $L$ be a finite lattice with $A(L)=\{a, b\}$ and $a=x_{0}<x_{1}<x_{2}<x_{3}<\cdots<x_{n} \quad$ and $b=y_{0}<y_{1}<y_{2}<$ $y_{3}<\cdots<y_{p}$. Then,
(1) $B=(a \vee b] \subseteq\left(x_{1} \vee y_{1}\right]$
(2) $\operatorname{gr}(\Gamma(L))=\operatorname{gr}\left(\Gamma\left(\left[x_{1} \vee y_{1}\right] \downarrow\right)=4\right.$
(3) $\operatorname{gr}(\Gamma(B))=\infty$

Example 2. Let $L=\{1,2,4,8,3,0\}$ be a lattice under the divisibility relation and 1 is the least and 0 is the largest element of $L$. Here, $B=\{1,2,3,0\}$ is the unique ideal, and $B_{1}=\{1,2,4,3,0\}$ is not since 3 is the complement of 2 and 4 .

Example 3. Let $A=\{2 k: \operatorname{gcd}(2 k, 3)=1\}$ for positive integer $k$ and let $L=A \cup\{0,1,3\}$. Then, $L$ is a lattice under divisibility relation and 1 is the least and 0 is the largest element of $L$. Here, $B=\{1,2,3,0\}$ is the unique ideal and $B_{1}=\{1,2,4,3,0\}$ and $B_{2}=\{1,2,4,8,3,0\} \ldots$ are not since 3 is the complement of more than two elements.
Proposition 3. Let $L$ be a lattice with the least element 0. Let $A(L)=\{a, b\}$ with a unique ideal $B=\left[1_{J}\right] \downarrow$. Then, $\Gamma(B)=K_{1,1}$.

Proof. By Lemma 5.6 of [2], $\Gamma(B)=K_{r, s}$. where $r$ is the number of elements in a set $([a] \uparrow \backslash[b] \uparrow)$ and $s$ is the number of elements in a set $([b] \uparrow \backslash[a] \uparrow)$. Now $[a] \uparrow=\{a, a \vee b\}$ and $[b] \uparrow=\{b, a \vee b\}, \quad([a] \uparrow \backslash[b] \uparrow)=\{a\}$. Similarly, $\quad([b] \uparrow \backslash$ $[a] \uparrow)=\{b\}$.

Proposition 4. Let $L$ be a lattice with the least element 0 and let $A(L)=\left\{a_{1}, a_{2}, a_{3}, \ldots, a_{n}\right\}$. Then, a unique sublattice $B=$ $\left[1_{J}\right] \downarrow$ is isomorphic to a Boolean ring $A$.

Proof. Let $B$ be the unique ideal of $L$. Then, by Definition 4.1 and Proposition 4.2 of $[2], \Gamma(B)=G(B)$, where $B$ is a Boolean ring under the operations $p+q=\left(p \wedge q^{\prime}\right) \vee\left(p^{\prime} \wedge q\right)$ and $p \cdot q=p \wedge q\left(p^{\prime}\right.$ is a complement of $p$ ).

Proposition 5. Let $L_{i}$ be the lattices with the least element $0_{i}: 1 \leq i \leq k$. Then, $L_{1} \otimes L_{2} \otimes L_{3} \otimes \cdots \otimes L_{k}$ is a lattice.

Proposition 6. Let $L_{i}$ be the lattices with the least element $0_{i}: 1 \leq i \leq k ; n_{i}=\left|A\left(L_{i}\right)\right|$. Then, $\left|A\left(L_{1} \otimes L_{2} \otimes L_{3} \otimes \cdots \otimes L_{k}\right)\right|=$ $n_{1}+n_{2}+n_{3}+\cdots+n_{k}$.

Proof. An ordered $k$-tuple $\left(b_{1}, b_{2}, b_{3}, \ldots, b_{k-1}, b_{k}\right)$ is an atom if at most one component is an atom in $A\left(L_{i}\right)$ and the rest of the components are corresponding zero's of $L_{i}$. Hence, the result.

Proposition 7. Let $L$ be a lattice with the least element 0 and $B$ be its unique ideal. Let $A(L)=\left\{a_{1}, a_{2}, \ldots, a_{n}\right\}$ be the set of atoms in $L$. Then, $\operatorname{cl}(\Gamma(B))=n$.

Proof. Suppose that $C=\left\{a_{1}, a_{2}, \ldots, a_{n}, b\right\}$ is a clique. Then, by definition of a unique ideal $B, b=a_{i} \vee a_{j}$ for some $i \neq j$, and $b \wedge a_{i} \neq 0$, shows that $C$ is not a clique.

Theorem 7. Let $L$ be lattice with the element 0 and having a finite set of atoms. Let $B$ be its unique ideal. Then, $|A(L)|=$ $n$ if and only if $\operatorname{cl}(\Gamma(B))=\chi(\Gamma(B))=n$.

Proof.

Case 1: clearly $\operatorname{cl}(\Gamma(B)) \leq \chi(\Gamma(B))$. Let $A(L)=\left\{a_{1}\right.$, $\left.a_{2}, \ldots, a_{n}\right\}$ be the set of atoms in $L$. We decompose $Z(B)^{*}$ as $P_{1}=B_{1}, P_{k}=\left\{x \in B_{k}: x \notin \underset{j<k}{\cup} B_{j}\right\}$ for $k=$ $2,3,4, \ldots, n$ and $B_{j}=\left\{x \in Z(B)^{*}: x \geq a_{j}\right\}$. Define a map $g$ on $Z(B)^{*}$ by $g(x)=k$ for $x \in P_{k}$. If $x, y$ are adjacent, then $g(x) \neq g(y)$, so $g$ is a coloring on $\Gamma(B)$; hence, $\chi(\Gamma(B) \leq n$ by Proposition 7. Conversely, assume that $A(B)$ is the set of atoms of $B$ is a clique in $B$. Then, $\operatorname{cl}(\Gamma(B))=n=|A(L)|$.

Case 2: if $B$ has an infinite number of atoms, then $c l(\Gamma(B))=\infty$; $\quad$ hence, $\quad \chi(\Gamma(B))=\infty$. Suppose 
$c l(\Gamma(B))=\infty=c l(\Gamma(L))$. Let $\left\{c_{1}, c_{2}, \ldots\right\}$ be an infinite clique in $\Gamma(B)$. Since $B$ is atomic, for each $i$, there exists an atom $a_{i}$ such that $a_{i} \leq c_{i}$. Again $c_{i}, c_{j}$ are adjacent for $i \neq j$, and it shows that $a_{i} \not c_{j}$. Hence, $B$ has an infinite number of atoms.

Proposition 8. Let $L$ be a lattice with the least element 0 and $B$ be its unique ideal. Then, $\chi(\Gamma(B))=\chi(\Gamma(L))$.

Proof. By Theorem 7, $X(T(B))=n$, applying Theorem 7 , for atomic lattice with n number of atoms, $X(T(L))=n$, similar to Theorem 2.1 in [1]. Following example shows that for nonatomic lattice $L$ with the least element 0 and at least two atoms, $\chi(\Gamma(B))=\chi(\Gamma(L))=2$.

Example 4. By Example 2 of [1], we know that the set $A=$ $\{1 / n: n$ is a positive integer $\} \cup\{0\}$ is a bounded distributive lattice having no atom. Let $D_{2}=\{1,2,5,10\}$ be a lattice of divisors of 10 under the divisibility relation. Then, $A \otimes D_{2}$ is nonatomic lattice with two atoms. The unique ideal $B=((0,2) \vee(0,5)]$. Here, $\chi(\Gamma(B))=\chi(\Gamma(L))=2$.

Lemma 4. Let $L$ be a lattice with the least element 0 and $B$ be its unique ideal. If $B$ contains an infinite chain, then $c l(\Gamma(L))=\infty$.

Proof. Let $\left\{b_{1}<b_{2}<\cdots\right\}$ be an increasing chain in $B$. Set $x_{i}=b_{i+1} \wedge b_{i}^{\prime}$. If $x_{i}=0$, then by distributivity in $B$, we have $b_{i}=b_{i} \vee\left(b_{i+1} \wedge b_{i}\right)=b_{i+1}$, which contradicts the hypothesis. Suppose that $x_{i}=x_{j}: i<j$ and $i+1 \leq j$. Therefore, $b_{i+1} \leq b_{j}$ and $x_{j}=b_{j+1} \wedge b_{j}^{\prime}=b_{i+1} \wedge b_{i}^{\prime} \wedge b_{j}^{\prime}=0$, a contradiction. This shows that $x_{i}$ are distinct. Again $b_{i+1} \wedge b_{j}^{\prime}=0$ implies that $x_{i} \wedge x_{j}=0: i \neq j$. Hence, $\left\{x_{i}: i=1,2,3, \ldots\right\}$ is an infinite clique in $L$.

\section{Diamond Lattices}

An element $a \in L$ is called a coatom if $a<1$. A lattice $L$ is said to be a diamond lattice if every element $a \in L, a \notin\{0,1\}$ is an atom and coatom. A diamond with $n$ atoms is denoted by $D_{n}$.

Theorem 8. Let $D_{2}=\left\{0, a_{1}, a_{2}, 1\right\}$ be a diamond. Then, $B=$ $\left\{0, a_{1}, a_{2}, 1\right\}$ is a distributive and uniquely complemented ideal of $D_{2}$. Moreover, $\Gamma(B)=\Gamma\left(D_{2}\right)=K_{1,1}$.

Theorem 9. Let $D_{n}=\left\{0, a_{1}, a_{2}, \ldots, a_{n}, 1\right\}$, be a diamond lattice. Then,

(1) For $n=2, B=\left\{0, a_{1}, a_{2}, 1\right\}$ is a distributive and complemented ideal of $D_{n}$

(2) For $n \neq 1, K_{n}=\Gamma\left(D_{n}\right)$

(3) For $n \geq 3, \operatorname{gr}\left(\Gamma\left(D_{n}\right)=3\right.$
Theorem 10. Let $L=D_{2} \otimes D_{n}$, be a lattice. Then, $B=D_{2} \otimes D_{2}$ is a distributive and complemented ideal of $L$, which can be generalized.

\section{Some Combinatorial Results}

Theorem 11. If $L$ is a lattice which is nondiamond or nondecomposable into diamonds with the least element 0 and $A(L)=\left\{a_{1}, a_{2}, a_{3}, \ldots, a_{n}\right\}$, then $\operatorname{deg}\left(a_{1}\right)={ }^{n-1} C_{1}+{ }^{n-1} C_{2}$ $+{ }^{n-1} C_{3}+\cdots+{ }^{n-1} C_{n-1}$ in $\Gamma(B)$.

Proof. Let $A(L)=\left\{a_{1}, a_{2}, a_{3}, \ldots, a_{n}\right\}(n \geq 2) . \Gamma(B)=\Gamma\left(\left[1_{J}\right] \downarrow\right)$. Then, in a finite subgraph $\Gamma(B), a_{1}$ is adjacent to $n-1$ vertices; hence, contribution of $\operatorname{deg}\left(a_{1}\right)$ is ${ }^{n-1} C_{1}$. Again $a_{1}$ is adjacent to $a_{i} \vee a_{j}, i, j \neq 1$, and contribution of $\operatorname{deg}\left(a_{1}\right)$ for this pair is ${ }^{n-1} C_{2}$. Continuing in this way, contribution of $\operatorname{deg}\left(a_{1}\right)$ for $a_{2} \vee \cdots \vee a_{k-1} \vee a_{k} \vee \cdots \vee a_{n}$ is ${ }^{n-1} C_{n-1}$. Total contribution is $\operatorname{deg}\left(a_{1}\right)={ }^{n-1} C_{1}+{ }^{n-1} C_{2}+{ }^{n-1} C_{3}+{ }^{n-1}+\cdots+{ }^{n-1} C_{n-1}$.

Corollary 5. Let $L$ be a lattice with the least element 0 and $B$ be its unique ideal. Then, for the induced subgraph $\Gamma(B)$,

(1) $\Delta(\Gamma(B))=\operatorname{deg}\left(a_{1}\right): a_{1} \in A(B)$

(2) $\Delta(\Gamma(B)) \leq \Delta(\Gamma(L))$

Proposition 9. Let $L$ be a finite lattice with the least element 0 with $A(L)=\{a, b\}$ and $a=x_{0}<x_{1}<x_{2}<\cdots<x_{k}$. Then, $\Delta(\Gamma(L))=k+1$.

Proof. Here, $B\left(x_{i}\right)=\{a\}$; hence, $x_{i} \wedge b=0$ for $1 \leq i \leq k$.

Proposition 10. Let $L$ be a lattice with the least element 0 , $A(L)=\left\{a_{1}, a_{2}, a_{3}, \ldots, a_{n}\right\}$ set of atoms, and $B$ be the unique sublattice of $L$. Then, $\left|Z(B)^{*}\right|=2^{n}-2, n \geq 2$.

Proof. Let $A(L)=\left\{a_{1}, a_{2}, a_{3}, \ldots, a_{n}\right\}=A(B)$. For $a_{i}, 1 \leq i \leq n$, zero divisors, their contribution is ${ }^{n} C_{1}$. Also $a_{i} \vee a_{j}, i \neq j$ are zero divisors, and their contribution is ${ }^{n} C_{2}$. Continuing is this way, contribution of $a_{1} \vee a_{2} \vee \cdots \vee a_{k-1} \vee a_{k+1} \cdots \vee a_{n}$ is a set of zero divisors and is $n={ }^{n} C_{n-1}$.

Corollary 6. Let $L$ be a finite lattice with the least element 0 and $B$ be its unique ideal. Then, $\left|Z(L)^{*}\right| \geq 2^{n}-2$.

Proof. Proof follows by Corollary 2 and Proposition 10.

\section{Data Availability}

Data from previous studies were used to support this study. They are cited at relevant places within the text as references.

\section{Conflicts of Interest}

The authors declare that they have no conflicts of interest. 


\section{References}

[1] S. K. Nimbhorkar, M. P. Wasadikar, and M. M. Pawar, "Coloring of lattices," Maths Slovaca, vol. 60, no. 4, pp. 419-434, 2010.

[2] E. Estaji and K. Khashyarmanesh, "The zero divisor graph of lattice," Results in Mathematics, vol. 61, no. 1-2, pp. 1-11, 2012.

[3] T. T. Chelvam and S. Nithya, "A note on the zero divisor Graph of lattice," Journal of Transactions on Combinatorics, vol. 3, no. 3, pp. 51-59, 2014.

[4] D. F. Anderson and P. S. Livingston, "The zero-divisor graph of a commutative ring," Journal of Algebra, vol. 217, no. 2, pp. $434-447,1999$.

[5] D. D. Anderson and M. Naseer, "Beck's coloring of a commutative ring," Journal of Algebra, vol. 159, no. 2, pp. 500-514, 1993.

[6] I. Beck, "Coloring of commutative rings," Journal of Algebra, vol. 116, no. 1, pp. 208-226, 1988. 


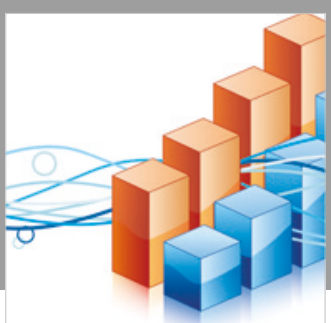

Advances in

Operations Research

\section{-n-m}
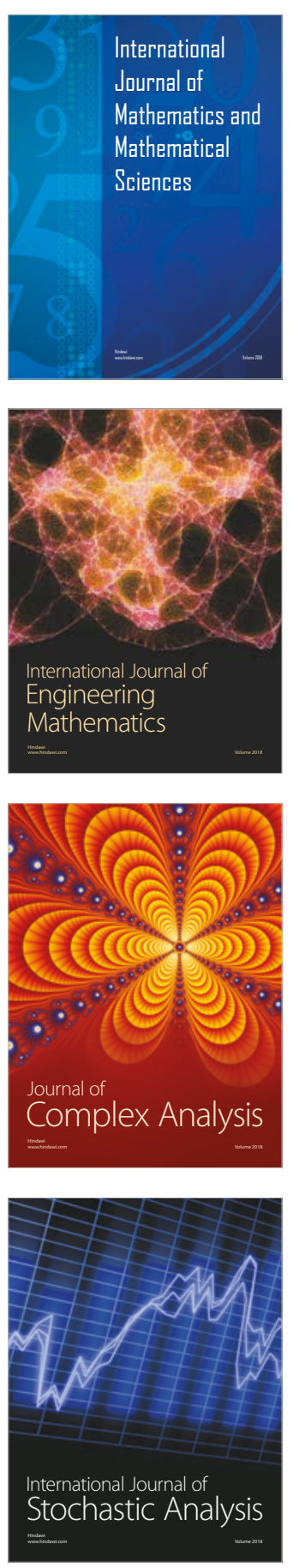
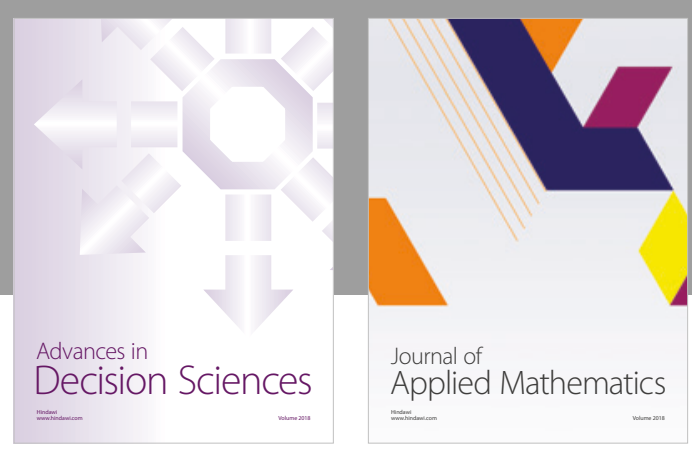

Journal of

Applied Mathematics
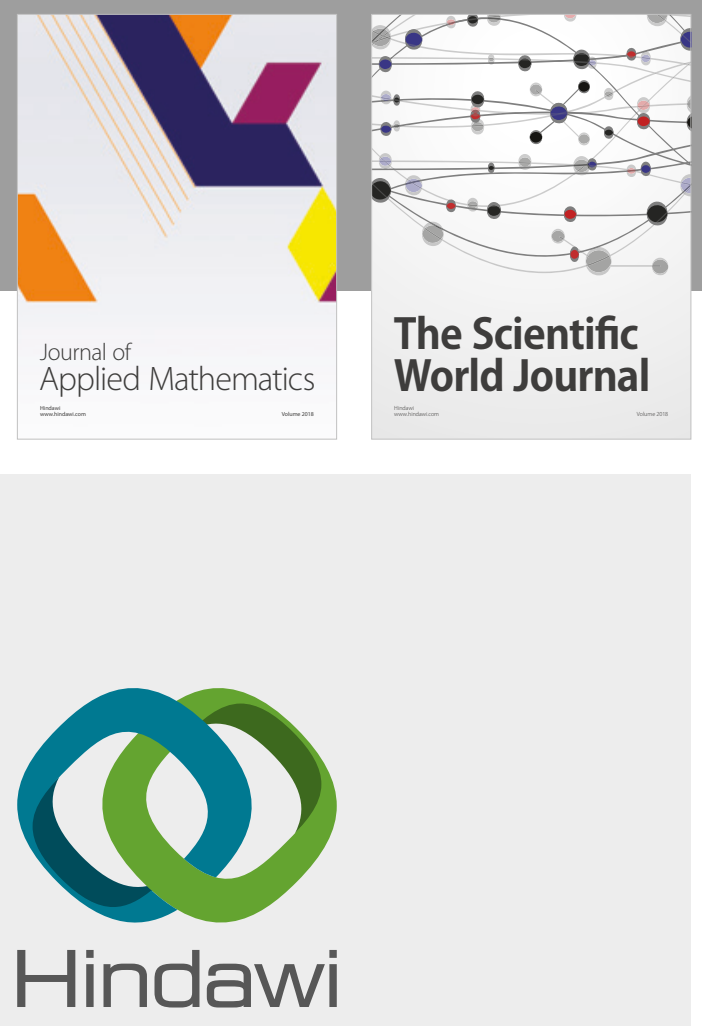

Submit your manuscripts at

www.hindawi.com

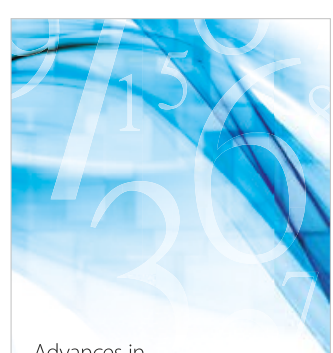

Advances in
Numerical Analysis
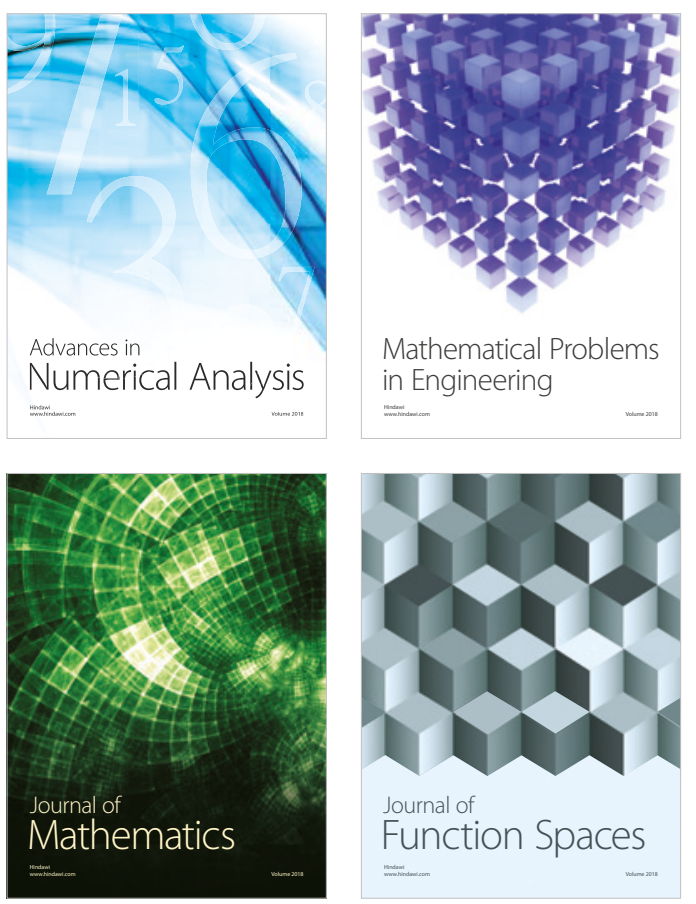

Mathematical Problems in Engineering

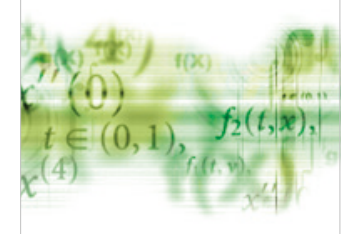

International Journal of

Differential Equations

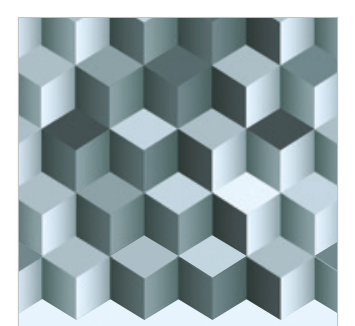

Journal of

Function Spaces

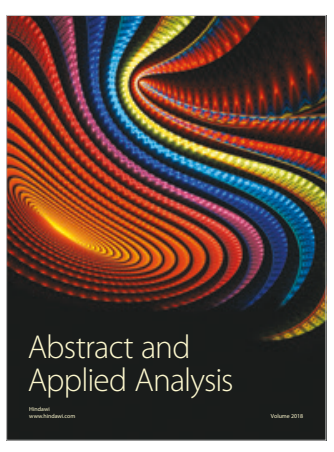

The Scientific

World Journal

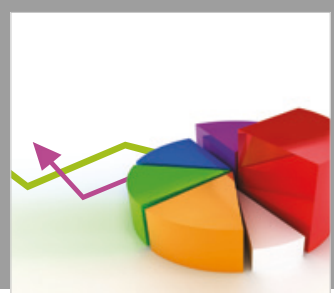

Journal of

Probability and Statistics
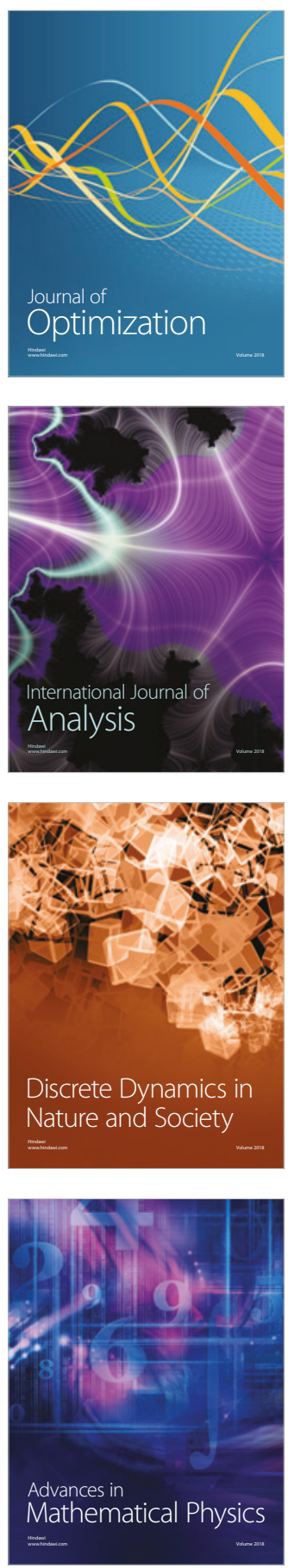